\title{
A study of maternal outcome in term premature rupture of membranes in a tertiary care hospital
}

\author{
Aayushi Jain*, Mohini Paul \\ Department of Obstetrics and Gynecology, Kasturba Hospital, Daryaganj, New Delhi, India \\ Received: 14 May 2020 \\ Accepted: 05 June 2020 \\ *Correspondence: \\ Dr. Aayushi Jain, \\ E-mail: jainaayushi88@gmail.com \\ Copyright: $\odot$ (the author(s), publisher and licensee Medip Academy. This is an open-access article distributed under \\ the terms of the Creative Commons Attribution Non-Commercial License, which permits unrestricted non-commercial \\ use, distribution, and reproduction in any medium, provided the original work is properly cited.
}

\begin{abstract}
Background: Premature rupture of membranes (PROM) remains a subject of great clinical relevance. The present study was conducted to study maternal morbidity and its relationship with PROM-delivery interval in patients with term PROM as compared to patients without PROM.

Methods: A prospective case control study was conducted in the department of obstetrics and gynecology, Kasturba Hospital, New Delhi. 100 pregnant patients presenting to the labor room with features of PROM at term (POG $>37$ weeks) were taken as cases and 100 term pregnant women (age and parity matched) with intact membranes were taken as controls and compared in terms of maternal outcome.

Results: This study reported maternal morbidity rate of $21 \%$ in term PROM cases which was significantly higher than in control group (5\%). The major cause was febrile morbidity which occurred in $16 \%$ of cases indicating ascending infection. Other complications were abdominal and episiotomy wound infection (4\%), chorioamnionitis $(2 \%)$, postpartum hemorrhage $(2 \%)$ and puerperal sepsis $(1 \%)$. There was an increased rate of operative delivery in the case group (33\%) as compared to $18 \%$ in patients without PROM. Duration of PROM-delivery interval had a significant direct proportional impact on the maternal morbidity. The duration of combined hospital stay of mother and neonate was also increased.

Conclusions: Patients with term PROM have significant maternal morbidity which was mainly due to infection. The duration of the hospital stay was also significantly increased.
\end{abstract}

Keywords: Chorioamnionitis, Lower segment caesarean section, Maternal morbidity, Postpartum hemorrhage, Term premature rupture of membranes

\section{INTRODUCTION}

Premature rupture of membranes (PROM) remains a subject of great clinical relevance for every obstetrician. Spontaneous rupture of membranes beyond 37 weeks of pregnancy but before onset of labor is called term premature rupture of membranes (PROM). The incidence of PROM is about $10 \%$ of all pregnancies and $70 \%$ of these occur at term. ${ }^{1}$ At term infection remains the most serious complication associated with PROM due to ascending infection from the vagina and cervix which can cause increased maternal morbidity. Various maternal complications include chorioamnionitis, abruptio placenta, febrile morbidity, failed induction, increased incidence of operative delivery, postpartum hemorrhage, retained placenta, puerperal sepsis, and endometritis. ${ }^{2-6}$

\section{METHODS}

A prospective case control study was conducted in the department of obstetrics and gynecology, Kasturba Hospital, Daryaganj, New Delhi after the approval of Institutional Review Board (IRB). Informed consent was taken by all the patients. In this study, 100 women with singleton pregnancy and cephalic presentation at term (gestational age >37 weeks) with features of pre-labor 
rupture of membranes presenting to the labor room of Kasturba Hospital were taken as cases. These were compared with 100 controls (normal term pregnancies without PROM matched to cases with respect to age and parity) in terms of maternal outcome. PROM was confirmed by fern test and $\mathrm{pH}$ paper test of clear leaking fluid on per speculum examination.

\section{Inclusion criteria}

- Cervical dilatation of less than $3 \mathrm{~cm}$ and lack of uterine contractions for at least 1 hour from the onset of PROM.

\section{Exclusion criteria}

- Gestational age less than 37 weeks, cervical dilatation more than $3 \mathrm{~cm}$, previous caesarean section, labor within 1 hour of rupture of membrane, malpresentations, multiple gestation and cephalopelvic disproportion.

Patients with term PROM were given prophylactic antibiotics and depending upon the Bishop's score, labor was induced with prostaglandin E2 gel or oxytocin. Time of induction was noted. The progress of labor in each case was closely monitored and plotted on a partograph. Antepartum, signs and symptoms of chorioamnionitis were observed. Postpartum, they were observed for third stage complications like postpartum hemorrhage and retained placenta. They were followed till puerperal period. Episiotomy wound and caesarean section wound were observed, and regular follow-up was done. Maternal morbidity in terms of puerperal fever, puerperal sepsis and wound infection were noted. Total hospital stay was calculated and compared in both the groups.

\section{Statistical analysis}

Suitable tests of significance were applied and p-values less than 0.05 was considered significant.

\section{RESULTS}

The results showed that maternal morbidity was significantly higher in term PROM patients compared with patients without PROM. Out of 100 cases, 21 cases had complications in their antepartum, intrapartum and postpartum period as compared to only 5 patients in control group. These results were statistically significant $(\mathrm{p}=0.001)($ Table 1$)$.

Table 1: Distribution of study subjects according to maternal morbidity.

\begin{tabular}{|llll|}
\hline Maternal morbidity & Cases & Controls & Total \\
\hline Absent & $79(79.00 \%)$ & $95(95.00 \%)$ & $174(87.00 \%)$ \\
\hline Present & $21(21.00 \%)$ & $5(5.00 \%)$ & $26(13.00 \%)$ \\
\hline Total & $100(100.00 \%)$ & $100(100.00 \%)$ & $200(100.00 \%)$ \\
\hline
\end{tabular}

$\left(\chi^{2}=11.317, \mathrm{df}=1\right)(\mathrm{p}$-value $=0.001)$

Table 2: Distribution of study subjects according to mode of delivery.

\begin{tabular}{|llll|}
\hline Mode of delivery & Cases & Controls & Total \\
\hline Lower segment caesarean section & $33(33.00 \%)$ & $13(13.00 \%)$ & $46(23.00 \%)$ \\
\hline Normal vaginal delivery & $67(67.00 \%)$ & $87(87.00 \%)$ & $154(77.00 \%)$ \\
\hline Total & $100(100.00 \%)$ & $100(100.00 \%)$ & $200(100.00 \%)$ \\
\hline
\end{tabular}

$\left(\chi^{2}=11.293, \mathrm{df}=1\right)(\mathrm{p}$-value $=0.001)$.

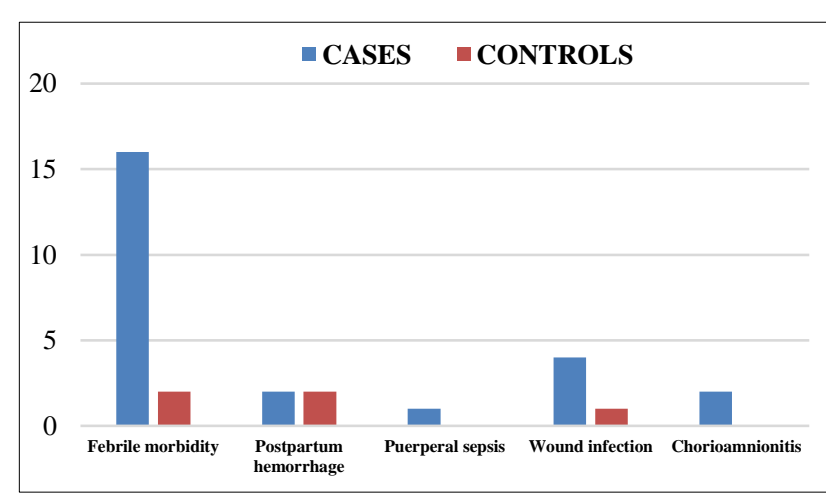

Figure 1: Distribution of study subjects according to the type of maternal complications.

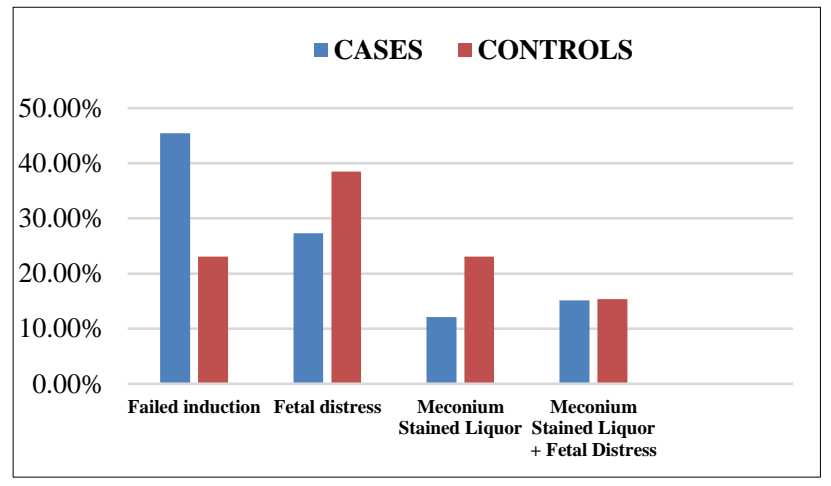

Figure 2: Indications of caesarean section. 
Most common cause of maternal morbidity in case group was febrile morbidity which occurred in $16 \%$ of cases indicating infection. Other complications were abdominal and episiotomy wound infection $(4 \%)$, chorioamnionitis $(2 \%)$, postpartum hemorrhage $(2 \%)$ and puerperal sepsis (1\%) (Figure 1).

There was significantly higher number of term PROM patients who underwent operative delivery $(\mathrm{p}=0.001)$. About $33 \%$ of women with term PROM underwent Lower segment caesarean section (LSCS) as compared to only $13 \%$ of women in control group. Whereas, $67 \%$ of women delivered vaginally in PROM group as compared to $87 \%$ in control group (Table 2).

In the term PROM patients, out of 33 patients, 15 patients underwent LSCS due to failed induction, 9 due to fetal distress, 4 due to meconium stained liquor and 5 had both meconium stained liquor with fetal distress. In the control group, only 3 out of 18 patients underwent LSCS due to failed induction, 5 due to fetal distress, 3 due to meconium stained liquor and 2 for meconium stained liquor with fetal distress (Figure 2).

\section{Table 3: Relationship between PROM-delivery} interval and maternal morbidity.

\begin{tabular}{|ll|}
\hline $\begin{array}{l}\text { PROM to delivery interval } \\
\text { (hours) }\end{array}$ & $\begin{array}{l}\text { Maternal } \\
\text { morbidity }\end{array}$ \\
\hline $0-6$ & $0(0.00 \%)$ \\
\hline $6.1-12$ & $0(0.00 \%)$ \\
\hline $12.1-24$ & $8(15.09 \%)$ \\
\hline$>24$ & $13(50.00 \%)$ \\
\hline Total & $21(21.00 \%)$ \\
\hline
\end{tabular}

The duration of PROM to delivery interval had statistically significant impact on maternal morbidity. Longer the PROM to delivery interval, higher chances of maternal complications. $50.00 \%$ of mothers had complications if the interval was more than 24 hours (Table 3).

Table 4: Distribution of study subjects according to hospital stay.

\begin{tabular}{|ll|}
\hline Mean hospital stay & Days \\
\hline Cases & $4.20 \pm 3.73$ \\
\hline Controls & $2.72 \pm 2.90$ \\
\hline
\end{tabular}

( $\mathrm{p}$ value $=0.002)$.

Mean hospital stay of cases was significantly higher $(4.20 \pm 3.73$ days) in patients with term PROM which included combined stay of mother and neonate. In control group mean hospital stay was $2.72 \pm 2.90$ days. The difference was statistically significant (Table 4).

\section{DISCUSSION}

\section{Maternal morbidity}

The incidence of maternal morbidity in this study was $21 \%$ in the case study group compared to only $5 \%$ in the control group.

The incidence of maternal morbidity was $14 \%$ in the study by Bangal $\mathrm{V}$ et al. ${ }^{8}$ Results similar to this study were seen in the study by Kodkany et al where $21 \%$ mothers had complications. ${ }^{7}$ This study results were comparable to studies by Singhal $\mathrm{P}$ et al, Devi A et al, Kodkany et al (Table 5). ${ }^{3,5,7}$

Table 5: Comparison of incidence of maternal morbidity in patients with term PROM across various studies.

\begin{tabular}{|c|c|c|c|c|}
\hline Study & Devi $A$ et al $^{3}$ & Singhal $\mathbf{P}$ et $\mathbf{a l}^{5}$ & Kodkany et al ${ }^{7}$ & Present study \\
\hline Incidence of maternal morbidity & $22.11 \%$ & $19 \%$ & $21 \%$ & $21 \%$ \\
\hline
\end{tabular}

Maternal morbidity is due to intrapartum infection which results from spread of ascending genital infection to amniotic cavity due to absence of the protective barrier of amniotic membranes in cases of PROM.

\section{Type of maternal complications}

Maternal outcome was measured in terms of chorioamnionitis, abruption, labor dystocia, postpartum hemorrhage, retained placenta, puerperal fever, delayed wound healing and wound infection.

Most common cause of maternal morbidity was febrile morbidity seen in $16 \%$ of cases in PROM group as compared to only $2 \%$ in control group, difference being statistically significant.
Similar results were reported by Devi A et al and Singhal $\mathrm{P}$ et al wherein study subjects had $20.19 \%$ and $17.5 \%$ febrile morbidity respectively. ${ }^{3,5}$ Revathi $\mathrm{V}$ et al noted $22 \%$ mothers with term PROM had puerperal fever. ${ }^{6}$ Lalwani A et al at reported $5.3 \%$ incidence of febrile morbidity in term PROM. ${ }^{2}$ Kadikar et al, reported $2 \%$ cases having puerperal fever. ${ }^{4}$ Wound infection was seen in $4 \%$ cases as compared to only $1 \%$ in the control group. Wound infection in terms of episiotomy and abdominal wound infection gaping was reported. $2.3 \%$ of patients had wound infection in the study by Lalwani A et al. ${ }^{2}$ Revathi $\mathrm{V}$ et al noted $14 \%$ incidence of wound infection. ${ }^{6}$ Wound infection rates in this study corroborated with the study by Kadikar et al study $(\sim 3 \%) .{ }^{4}$ Chorioamnionitis is an important and peculiar sequalae of PROM. It is a grave complication of PROM where the mother has to be 
treated aggressively with broad spectrum antibiotics and pregnancy has to be terminated immediately.

It was seen in 2 cases in term PROM group in this study whereas none of the patients had chorioamnionitis in the control group. This study results showed much lower rate of chorioamnionitis than compared to the study of Devi A et al, Kodkany et al and Pandey S et al probably because all patients with features of PROM at this hospital were given prophylactic intravenous antibiotics (Table 6). , $^{3,9,10}$

Table 6: Comparison of incidence of chorioamnionitis in patients with term PROM across various studies.

\begin{tabular}{|lllll|}
\hline Study & Pandey S et al ${ }^{9}$ & Kodkany et al $^{7}$ & Devi A et al $^{3}$ & Present study $^{2}$ \\
\hline Incidence of chorioamnionitis & $6 \%$ & $5 \%$ & $5.6 \%$ & $2 \%$ \\
\hline
\end{tabular}

Postpartum hemorrhage occurred in $2 \%$ cases in this study. One patient out of them was a grand multipara and had atonic PPH. Results were similar to study by Lalwani A et al who noted $2.3 \%$ patients with term PROM had PPH. ${ }^{2}$ One patient on $5^{\text {th }}$ postoperative day of LSCS developed high grade fever with chills and foul-smelling lochia. She had history of leaking for more than 24 hours and her high vaginal swab antenatally was positive for Group B Streptococcus. She was treated aggressively with broad spectrum antibiotics and her wound gaped on $10^{\text {th }}$ day. She underwent abdominal wound resuturing and was discharged on $15^{\text {th }}$ postoperative day. Hence, if early intervention and steps for termination of pregnancy are taken it will lead to decreased morbidity of mothers. Maternal health is the primary indicator for the need to deliver. Any evidence of infection or maternal instability due to complications of PROM requires careful evaluation and determination of the appropriate management.

\section{Mode of delivery}

In this study, 33\% of women with term PROM underwent lower segment caesarean section (LSCS) as compared to only $13 \%$ of women in control group. Whereas, $67 \%$ of women delivered vaginally in PROM group as compared to $87 \%$ in control group. The results were statistically significant indicating higher rate of caesarean section in PROM group in this study.

Vaishnav $\mathbf{J}$ et al, found no correlation in mode of delivery and PROM. ${ }^{11}$ They noted $21.21 \%$ LSCS rate in cases and $18.18 \%$ LSCS rate in controls. Revathi V et al, in a study noted a fourfold higher caesarean section rate in term PROM cases, the rate being $29 \% .^{6}$

Comparable results were noted by Shrestha SR et al i.e., $27 \%$ and $30 \%$ in Kodkany et al. ${ }^{7,10}$ Kadikar et al observed that in term PROM, 33 cases had vaginal delivery (48\%), 28 cases had cesarean section $(41 \%)$ and $8(11 \%)$ cases had instrumental delivery. ${ }^{4}$ This study results were comparable to study of Revathi V et al, Shrestha SR et al, Kodkany et al and Kadikar et al (Table 7). 4,6,7,10

Higher rates of caesarean section were mainly observed in patients with PROM due to immediate induction given to these patients which resulted in increased operative delivery.

Table 7: Comparison of mode of delivery (normal vaginal delivery versus lower segment caesarean section) in patients with term PROM across various studies.

\begin{tabular}{|c|c|c|c|c|c|}
\hline Study & 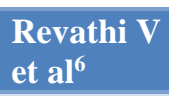 & $\begin{array}{l}\text { Shrestha } \\
\text { SR et al } \mathbf{l}^{10}\end{array}$ & $\begin{array}{l}\text { Kodkany } \\
\text { et } \text { al }^{7}\end{array}$ & $\begin{array}{l}\text { Kadikar } \\
\text { et } \mathbf{~ a l ~}^{4}\end{array}$ & Present study \\
\hline Normal vaginal delivery & $61 \%$ & $70 \%$ & $68 \%$ & $48 \%$ & $67 \%$ \\
\hline Lower section caesarean section & $29 \%$ & $27 \%$ & $30 \%$ & $41 \%$ & $33 \%$ \\
\hline
\end{tabular}

\section{Relationship between maternal morbidity and PROM- delivery interval}

The duration of PROM to delivery interval had statistically significant impact on maternal morbidity. Longer the PROM to delivery interval, higher chances of maternal morbidity. $50.00 \%$ of mothers had complications if the interval was more than 24 hours compared to none if they delivered within 12 hours.
While it was $15.09 \%$ if this interval was in between 1224 hours.

Revathi $\mathrm{V}$ et al, reported maternal morbidity of $20.68 \%$ in 12-24-hour interval which increased to $75.86 \%$ in more than 24 hours PROM to delivery interval. ${ }^{6}$

Rakholia $\mathrm{S}$ et al also reported $0 \%, 12.5 \%$ and $75 \%$ maternal morbidity in PROM to delivery interval 0-12 
hour, 12-24 hour and more than 24 hour respectively. ${ }^{12}$ Bangal $\mathrm{V}$ et al observed $0 \%, 16 \%$ and $64 \%$ in PROM to delivery interval 0-12 hour, 12-24 hour and more than 24 hour respectively. ${ }^{8}$ This study was comparable to studies by Rakholia $\mathrm{S}$ et al and Bangal V et al (Table 8). ${ }^{8,12}$

Table 8: Comparison of PROM to delivery interval with maternal morbidity in patients with term PROM across various studies.

\begin{tabular}{|c|c|c|c|c|}
\hline Study & & Rakholia S et al ${ }^{12}$ & Bangal $\mathrm{V}$ et $\mathbf{a l}^{8}$ & Present study \\
\hline \multirow{4}{*}{$\begin{array}{l}\text { PROM to delivery interval with maternal } \\
\text { morbidity }\end{array}$} & 0-6 hour & $0 \%$ & $0 \%$ & $0 \%$ \\
\hline & 6-12 hour & $0 \%$ & $0 \%$ & $0 \%$ \\
\hline & 12-24 hour & $12.5 \%$ & $16 \%$ & $15.09 \%$ \\
\hline & $>24$ hour & $75 \%$ & $64 \%$ & $50.00 \%$ \\
\hline
\end{tabular}

This lends credence to the observation that risk of infection and associated chorioamnionitis and thus morbidity increases with the passage of time after PROM. Thus, it seems prudent to induce labor immediately in PROM patients in order to shorten the PROM to delivery interval and the associated maternal morbidity.

\section{Mean hospital stay}

It was observed that mean hospital stay of patients with term PROM was significantly higher $(4.20 \pm 3.73$ days $)$ which included combined stay of mother and neonate. It was also majorly due to neonatal morbidity. It was mainly due to antibiotics given to baby. In the control group mean hospital stay was $2.72 \pm 2.90$ days. This is lower than the study by Shah $\mathrm{M}$ et al in which hospital stay in patients of term PROM was longer (5.98 days) as compared to control (3.96 days). ${ }^{1}$ Vaishnav $\mathrm{J}$ et al in a study also found higher hospital stay to the order of 6.87 days in case group which they attributed to antibiotics given to baby due to neonatal morbidity for which mother was kept in ward (Table 9). ${ }^{11}$

Table 9: Comparison of mean hospital stay in patients with term PROM across various studies.

\begin{tabular}{|lllll|}
\hline Study & & $\begin{array}{l}\text { Shah M } \\
\text { et al }^{\mathbf{1}}\end{array}$ & $\begin{array}{l}\text { Vaishnav } \\
\text { J et al }^{11}\end{array}$ & $\begin{array}{l}\text { Present } \\
\text { study }\end{array}$ \\
\hline $\begin{array}{l}\text { Mean } \\
\text { hospital } \\
\text { stay } \\
\text { (days) }\end{array}$ & Cases & 5.98 & 6.87 & $4.20 \pm 3.73$ \\
\cline { 2 - 5 } & Controls & 3.96 & 4.36 & $2.72 \pm 2.90$ \\
\hline
\end{tabular}

\section{CONCLUSION}

Term PROM is an important complication of pregnancy which leads to significant maternal morbidity. Pregnancy complicated with PROM can led to complications such as chorioamnionitis, postpartum hemorrhage, puerperal fever, puerperal sepsis and delayed wound healing, wound infection. Also, it leads to increased operative interventions leading to higher rates of operative delivery. These complications can be reduced by use of appropriate antibiotics and immediate induction of labor. Health care strategies should strive to decrease and eliminate genital tract infections with the help of a low-cost tool such as syndromic approach. By making antenatal care and basic health services available to women of all sections of society the incidence of PROM and associated maternal morbidity can be reduced.

\section{Funding: No funding sources}

Conflict of interest: None declared

Ethical approval: The study was approved by the Institutional Ethics Committee

\section{REFERENCES}

1. Shah M, Sandesara P. Fetomaternal outcome in cases of PROM. Gujarat Med J. 2011;66:1.

2. Lalwani A, Najam R. The Prospective study of the incidence, etiological factors and maternal outcome in pre-labour ruptured membranes (term and pre-term). Ann Int Med Den Res. 2016;2(2):121-25.

3. Anjana D, Reddi R. Premature rupture of membrane: A clinical study. J Obst Gynecol India. 1996;46:63-76.

4. Kadikar GK, Gandhi MR, Damani SK. A study of fetomaternal outcome in cases of premature rupture of membrane. IJSR. 2014;3(3):299-301.

5. Singhal P, Singhal AK. Fetomaternal outcome in premature rupture of membranes. Obst Gynae Today. 2002;10:585.

6. Revathi V, Sowjanya R, Lavanya S. Maternal and perinatal outcome in premature rupture of membranes at term. IOSR-JDMS. 2015;14:12-5.

7. Kodkany, Telang. Premature rupture of membranes, a study of 100 cases. J Obstet Gynecol India. 1991;41:492.

8. Bangal VB, Gulati P, Shinde KK, Borawake SK. Induction of labour versus expectant management for premature rupture of membranes at term. Int J Biomed Res. 2012;3:164-70.

9. Pandey S, Dave A, Bandi S. Maternal and fetal outcome in cases of prom. J Obstet Gynecol India. 2000;50:63.

10. Shrestha SR, Sharma P. Fetal outcome of pre-labor rupture of membranes. Nepal J Obstet Gynaecol. 2006;1(2):19-24.

11. Vaishnav J, Vaishnav G. A study of foeto-maternal outcome in patients with prelabour rupture of 
membranes at term $(>37)$ weeks. Med Sci. 2012;1(2):118-24.

12. Rakholia S. Study of feto-maternal outcome of delayed intervention in cases of term premature rupture of membrane. Glob J Res Anal. 2016;5(1):149-51.
Cite this article as: Jain A, Paul M. A study of maternal outcome in term premature rupture of membranes in a tertiary care hospital. Int J Reprod Contracept Obstet Gynecol 2020;9:2953-8. 